\title{
Comparison of ASIM Traffic Profile Detectors and Floating Car Data During Traffic Incidents
}

\author{
Lukáš Rapant $^{1}$, Kateřina Slaninová ${ }^{1}$, Jan Martinovič ${ }^{1}$, Marek Ščerba ${ }^{2}$, and \\ Martin Hájek ${ }^{1}$ \\ 1 VŠB - Technical University of Ostrava, \\ IT4Innovations, \\ 17. listopadu 15/2172, Ostrava, Czech Republic \\ 2 Transport Research Centre, \\ Líšeňská 33a, Brno, Czech Republic \\ $\{$ jan.martinovic, katerina.slaninova, lukas.rapant, martin.hajek\}@vsb.cz, \\ marek.scerba@cdv.cz
}

\begin{abstract}
Intelligent Transportation Systems are highly dependent on the quality and quantity of road traffic data. The complexity of input data is often crucial for effectiveness and sufficient reliability of such systems. Recent days, the fusion of various data sources is the topic which attracts attention of several researchers. The algorithms for data fusion take benefit of the advantages and disadvantages of each technology, resulting in an optimal solution for traffic management problems. The paper is focused on finding relations between two main data sources, floating car data and ASIM traffic profile detectors. Time series of speed and other information obtained from these data sources were analysed by Granger causality with intention to use both data sources efficiently for traffic monitoring and control during traffic incidents.
\end{abstract}

Keywords: traffic analysis, Granger causality test, traffic incident detection, floating car data, ASIM sensors

\section{Introduction}

Intelligent Transportation Systems (ITS) are highly dependant on the quality and quantity of road traffic data. The complexity of input data is often crucial for effectiveness and sufficient reliability of such systems. Recent days, the fusion of various data sources is under the development and new algorithms for data fusion are discussed. Common data input is traffic information such as vehicle speed or traffic flow collected through fixed detectors placed along the road network at strategic points. Other valuable data source based on collecting traffic data through mobile devices and on-board units has become important as a source that can provide accurate real-time information over a large road network and overcomes some problems related to fixed detectors.

The floating car data (FCD) technique is based on the exchange of information between floating cars travelling on a road network and a central data 
system. The floating cars periodically send the recent accumulated data on their positions, whereas the central data system tracks the received data along the travelled routes. The frequency of sending/reporting is usually determined by the resolution of data required and the method of communication.

The most common and useful information that FCD techniques and ITS provide is average travel times and speeds along road links or paths $[7,17,11,16,6]$. They deploy FCD in order to predict short-term travel conditions, to automatically detect incident or critical situations [3,13,15], or determine OriginDestination traffic flow patterns $[12,10]$.

The reliability of all types of estimates based on FCD highly depends on the percentage of floating cars participating in the traffic flow. Several FCD systems were presented, integrating short-term traffic forecasting based on current and historical FCD. However, these systems exploit data mostly from car flotillas to deliver real-time traffic speed information throughout large cities, signalized urban arterials or particular parts of traffic network, for example Italian motorway [1], Berlin [8], Beijing [9], Vienna [5], and many others.

The RODOS Transport Systems Development Centre ${ }^{3}$ operates the system viaRODOS ${ }^{4}$ which covers the whole traffic network of the Czech Republic [2]. Therefore, we are able to monitor traffic situation from the global perspective. On the other hand, this globalisation brings us several restrictions. To cover the whole traffic network, the system viaRODOS uses segmentation system which divides the highways and speed ways onto smaller parts - segments. The location code table from Traffic Message Channel (TMC) - a technology for delivering traffic and travel information to drivers - is used for identification of real world objects localization. Each row in location code table is strongly connected to a specific geographical entity (crossroads, roads, important objects, etc.). Locations used by TMC system are set by the rules for location identification defined by the international standard EN ISO 14819-3:1999. The location code tables for Czech Republic is created by Central European Data Agency (CEDA), which also addresses their certification on the international level.

The viaRODOS system operates the complex database of people and goods mobility database integrating various types of data sources like traffic data consisted of data from toll gates electronic system and ASIM traffic detectors, floating car data, meteodata, and data from Uniform system of traffic information for the Czech Republic. The appropriate combination of all the data sources and their usage after suitable preprocessing allow us to obtain more accurate results for traffic monitoring and control and for short-time predictions.

FCD data are usually used for quality control of static sensor infrastructure like identifying faulty traffic detectors [5], for assessment of traffic message system quality [14], or for traffic state analysis to evaluate the effectiveness of traffic control measures (signalling optimization). The published contributions are focused on the exact position of the traffic detectors, mainly within urban

\footnotetext{
${ }^{3}$ RODOS Transport Systems Development Centre: http://www.it4i-rodos.cz/ defaultEN.aspx

4 viaRODOS is available on http://www.viarodos.eu
} 
networks. We do not have opportunity to influence the distribution of the traffic detectors, mainly due to its expensiveness, and have a set network of TMC segments to measure FCD data. However, using appropriate methods and algorithms, it is possible to obtain sufficient outputs for traffic management and short-time prediction.

The paper is focused on finding relations between time series obtained from ASIM traffic profile detectors and floating car data with intention to use both data sources for traffic monitoring and control during traffic incidents. The compared data sources are described in more detail in Section 2. The comparison was done by identifying Granger causality, see Section 3. The experimental results are then described in Section 4, and Section 5 concludes the paper.

\section{Data Sources}

Generally, data sources describing actual traffic situation on Czech motorways can be divided into two groups - stationary data sources and floating car data sources. Stationary data sources contain data provided by toll gates and the data provided by ASIM sensors. However, the value of the data from the toll gates is severely reduced by the fact, that they only contain information about large vehicles (trucks, buses, etc.). Data from toll gates thus only describes this specific part of the traffic, and is not usable for the description of general traffic situation. The following text briefly describes both ASIM sensor data and floating car data and summarizes their advantages and disadvantages.

\section{$2.1 \quad$ ASIM Sensors}

In the Czech Republic, the traffic situation is mostly monitored and evaluated using the stationary data. One of the most important source of stationary data is ASIM sensor network. ASIM sensors are placed on certain toll gates (all these toll gates are placed on the highways). They comprise of various sensors like passive infrared detectors and radars. They are able to distinguish individual vehicle types, and measure their speed and intensity. Their measurements are aggregated every five minutes and mean speed and intensity are calculated.

They have number of advantages. One of the biggest advantages is the fact that there is no need for equipping vehicles with additional electronic devices. Consequently, speeds of all vehicles going through a sensor are measured. Another important advantage is detail of the data. ASIM sensors provide separate information about every lane of the monitored road. Moreover, since the ASIM sensor is able to distinguish type of the passing vehicle, it is only data source which is able to provide speeds and intensities for each type of the vehicle.

There are, however some serious disadvantages of these sensors. In the Czech Republic, this network of measuring points is very sparse. There are only about 120 toll gates equipped with ASIM sensors; all of these are placed on the motorways. This low density is caused by related necessary expenses - installation of such measuring points is quite expensive. There are also other limitations. 
Electronic toll gates divide roads into fragments of various length, some of them may extend to many kilometres. Thus, data obtained from ASIM sensors exactly describe only traffic situation around the tollgate.

\section{$2.2 \quad$ Floating Car Data}

The opposite to stationary data is Floating Car Data (FCD). This approach is based on the measurements of location, speed, travel direction and time information from certain vehicles in the traffic. These information are obtained from the GPS receiver inside the car and broadcast by radio unit or cell phone. Nowadays, an on-board unit including a GPS receiver becomes a standard equipment of corporate fleet cars. Moreover, increasing expansion of smart phones brought GNSS technology to our personal lives, where with combination of cheap connectivity, each vehicle can become a source of this type of data. FCD have specific discretization. For example, D1 highway is divided into the sections (TMC segments) with length from several hundred meters to few kilometres. The traffic speed is calculated each minute as a mean of speed of all floating cars that passed through the section in the last minute.

This approach again has several advantages and drawbacks. The number of cars equipped with a GPS unit has doubled over the past five years. It can be expected that the trend will continue. It implies that the number of potential data sources will increase. Moreover, data from GPS receivers is not limited to the predefined places so the coverage is much larger than in the case of the stationary data.

Disadvantages come mainly from the GNSS technology itself. GPS device as a part of GNSS technology, fails to provide precise outputs or the outputs can be intentionally distorted. The quality of outputs can also be influenced by many factors such as the device quality, location, weather or other unpredictable and uncontrollable phenomena. All of this can have an impact on positioning, ranging from meters to tens of meters. Because GNSS is based on satellite technology, GPS receiver has to be able to receive signals from several satellites. This can prove to be difficult in some cases. Typical example is an urban area with tall buildings which form obstacles between receiver and satellites. Then, GPS receiver is not able to report its position.

\section{Granger Causality}

A variable $x$ is said to Granger cause another variable $y$ if past values of $x$ help predict the current value of $y$. This definition is based on the concept of causal ordering [4]. Two variables can be correlated by chance but it is improbable that the past values of $x$ will be useful in predicting $y$, given all the past values of $y$, unless $x$ does in some way actually cause $y$. Granger causality is not identical to causation in the philosophical sense, but it does demonstrate the likelihood of such causation or the lack of such causation more forcefully than does simple correlation. For example, where the third variable drives both $x$ and $y, x$ might 
still appear to drive $y$ though there is no actual philosophical causal mechanism directly linking the variables. However, there can be still Granger causality as one variable may be useful for predicting the other. The simplest test of Granger causality requires estimating the following two regression equations:

$$
\begin{aligned}
& y_{t}=\gamma_{1,0}+\sum_{i=1}^{l} \gamma_{1, i} y_{t-i}+\sum_{j=1}^{l} \gamma_{1, l+j} x_{t-j}+\varepsilon_{t} \\
& x_{t}=\gamma_{2,0}+\sum_{i=1}^{l} \gamma_{2, i} x_{t-i}+\sum_{j=1}^{l} \gamma_{2, l+j} y_{t-j}+\varepsilon_{t}
\end{aligned}
$$

where 1 is the number of lags that adequately models the dynamic structure so that the coefficients of further lags of variables are not statistically significant and the error terms $\epsilon$ are white noise. Number of lags $l$ is usually chosen using an information criterion, in our case Bayesian information criterion. If the $l$ parameters $\gamma_{1, l+j}$ are jointly significant according to the F-test then the null hypothesis that $x$ does not Granger cause $y$ can be rejected. Similarly, if the $l$ parameters $\gamma_{2, i}$ are jointly significant according to the F-test, then the null hypothesis that $y$ does not Granger cause $x$ can be rejected. This test is Granger causality test.

\section{Experimental Results}

Two toll gates with ASIM sensors and their appropriate FCD segments were chosen for the experiments. The first toll gate (labeled 34.1) is placed on 195.7th $\mathrm{km}$ of D1 motorway in direction to Ostrava and the other (labeled 15.2) is placed on 117.7th km of D1 in direction to Prague. Lengths of their corresponding FCD segments are roughly $2 \mathrm{~km}$ in both cases and the gates are placed in the middle of the segments. These gates and segments were chosen because of higher frequency of traffic incidents in these locations. These locations are also interesting because the first one is located near the city and the other one is not. Data comes from period of March to April 2014.

As it was mentioned in the introduction, our main interest is in determination of relationship between FCD and ASIM data also during the traffic incidents, respectively, whether this relation is causal or not. In case of longer time series without any incidents it can be quite unsurprisingly shown that there exists a causal relationship. This causality in case of gate 34.1 and its corresponding segment can be seen in Figure 1.

From the perspective of Granger causality test, both relations are causal (i.e. ASIM contains significant information about FCD and vica versa). In case of ASIM time series causing FCD time series, test statistic has value of 51.82 with critical value of 2.7 and in case of FCD causing ASIM, value test statistic was 12.36 with critical value 2.3. Therefore, in both cases we can reject null hypothesis that there is no causality between the time series. Similar results were received in case of other sensors-segments and time periods. 


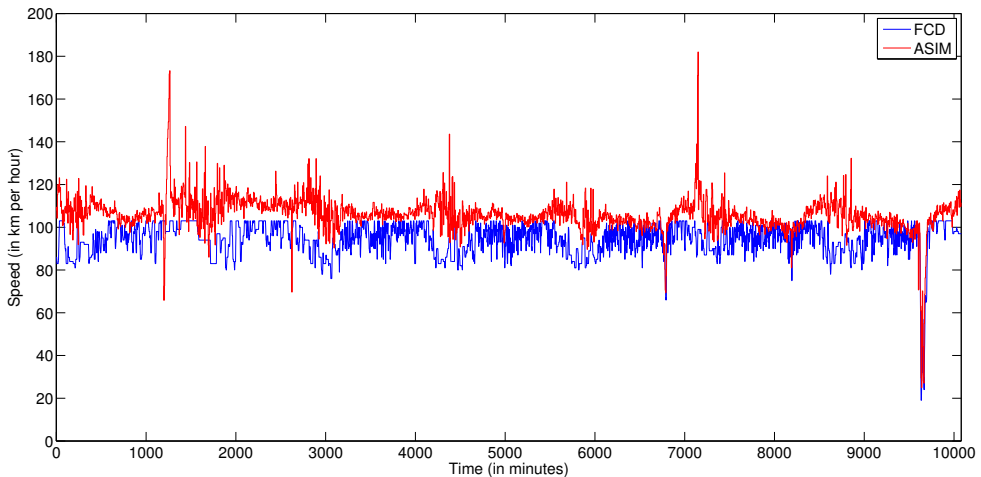

Fig. 1. Time series of speed from ASIM sensor 34.1 and corresponding FCD segment form the third week of march 2014

Based on this we can declare that ASIM time series can be used for completion and prediction of FCD series and vice versa. It would not be difficult as, most of the time, traffic is periodic and easily predictable. This is, however, of little use as the most interesting part of the traffic are the traffic incidents. It is quite possible that margin of error allowed by the Granger causality can come from these rare events. Therefore, it is important to know whether these causal relations work even in case of traffic incidents on shorter time series. There were 16 incidents detected by our method during observed period (six in case of sensor 34.1 and ten in case of sensor 15.2). Results from Granger causality tests performed on these incidents can be seen in Tables 1 and 2 ( $T$ is test statistic and $k$ is critical value; tests which have not confirmed causality are marked by red colour).

\begin{tabular}{|c|c|c|}
\hline Time of incident & $\begin{array}{c}\text { ASIM causes FCD } \\
{[T / k]}\end{array}$ & $\begin{array}{c}\text { FCD causes ASIM } \\
{[T / k]}\end{array}$ \\
\hline \hline 8. 3. $201414: 36-17: 20$ & $0.84 / 3.85$ & $3.69 / 3.87$ \\
\hline 21. 3. $201416: 19-17: 55$ & $6.49 / 3.89$ & $9.7 / 3.04$ \\
\hline 16. 4. $201416: 11-17: 21$ & $1.04 / 3.87$ & $17.34 / 3.87$ \\
\hline 18. 4. $201416: 49-18: 48$ & $0.51 / 3.87$ & $19.07 / 3.1$ \\
\hline 23. 4. $20149: 46-10: 27$ & $44.28 / 3.89$ & $7.17 / 3.04$ \\
\hline 29. 4. $201417: 00-18: 33$ & $18.51 / 3.87$ & $5.92 / 3.88$ \\
\hline
\end{tabular}

Table 1. ASIM sensor 34.1 causality

Three different kinds of outcome can be seen in Table 1 and Table 2. The first one is that both causality tests were passed. This is the most usual outcome and it implies that both ASIM data and FCD data react on the incident at roughly 


\begin{tabular}{|c|c|c|}
\hline Time of incident & $\begin{array}{c}\text { ASIM causes FCD } \\
{[T / k]}\end{array}$ & $\begin{array}{c}\text { FCD causes ASIM } \\
{[T / k]}\end{array}$ \\
\hline \hline 24. 3. $20149: 17-21: 05$ & $14.02 / 3.85$ & $21.75 / 3.85$ \\
\hline 25. 3. $20147: 13-12: 15$ & $5.14 / 3.02$ & $15.89 / 3.86$ \\
\hline 25. 3. 2014 17:05-21:07 & $16.73 / 3.01$ & $0.63 / 3.86$ \\
\hline 26. 3. 2014 9:01-10:16 & $1.55 / 3.87$ & $0.7 / 3.87$ \\
\hline 26. 3. 2014 14:13-15:05 & $0.16 / 3.92$ & $3.42 / 3.93$ \\
\hline 26. 3. $201415: 36-20: 17$ & $7.35 / 3.88$ & $3.03 / 3.02$ \\
\hline 28. 3. $201415: 55-19: 16$ & $0.88 / 3.86$ & $5.24 / 3.86$ \\
\hline 1. 4. 2014 16:05-16:46 & $8.41 / 3.89$ & $9.71 / 2.05$ \\
\hline 10. 4. 2014 16:40-17:33 & $14.07 / 3.87$ & $16.20 / 3.02$ \\
\hline 22. 4. 2014 7:30-9:56 & $12.89 / 2.40$ & $9.52 / 3.88$ \\
\hline
\end{tabular}

Table 2. ASIM sensor 15.2 causality

the same time. It probably means that incident happened somewhere near the toll gate with ASIM sensor. Such incident can be seen in Figure 2. The fact that this outcome represents more then a half of the detected incidents means, that most of the time, both of the sensors react well and at the similar time on the development of the traffic.

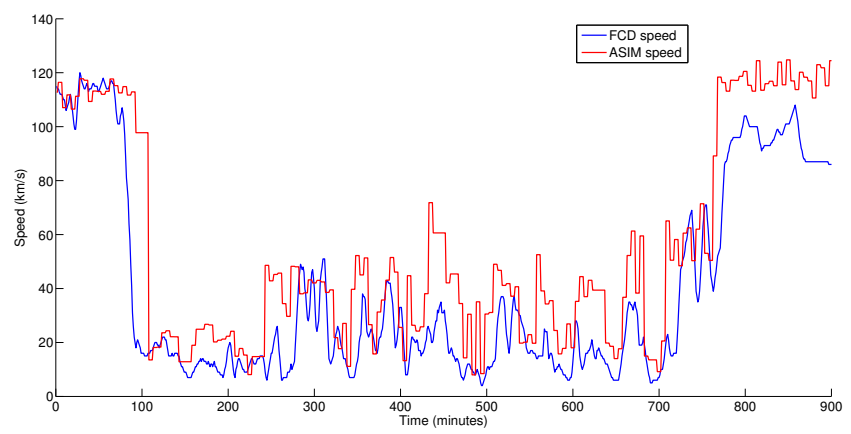

Fig. 2. Time series of speed from ASIM sensor 15.2 and corresponding FCD segment form 24. 3. 2014

The second possible outcome is that there is only one way causality between the time series. Such example is in Figure 3. It happens either when the incident is further from the toll gate with ASIM and is sooner registred by FCD or is located in the close vicinity of the ASIM sensor. Due to the sparsity of the ASIM sensor network (sensors on D1 are placed about $12 \mathrm{~km}$ apart), it is much more usual that FCD data detects the incident sooner than ASIM sensors and are therefore usable for the predictions of speeds in ASIM sensor time series. Opposite scenario is much rarer. If we take into account the fact that most of 
the time both series are roughly equivalent, we can assume that it is quite safe to predict ASIM speed time series from FCD time series. If we try it other way, there is greater possibility of failure due to the sensor sparsity.

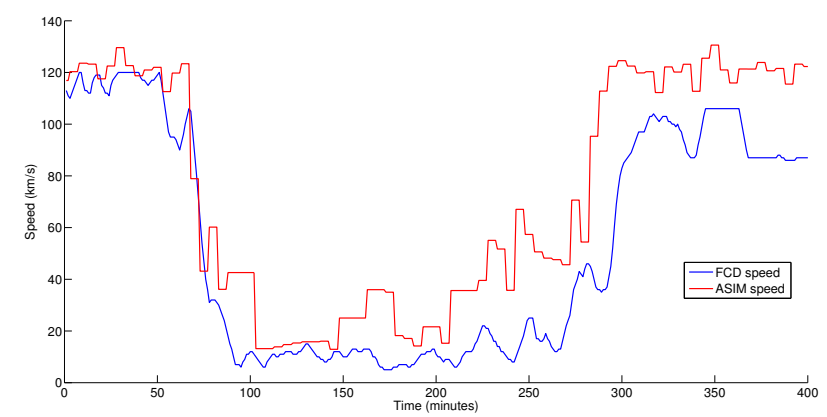

Fig. 3. Time series of speed from ASIM sensor 15.2 and corresponding FCD segment form 25. 3. 2014

The third possible outcome is that there is no causality either way. These outcomes prove to be problematic because they cannot be easily explained and can imply some problems regarding predictability. Because of this fact, they will be thoroughly analyzed.

The first of the three analysed incidents is the incident from 8.3.2014 from 195.7th $\mathrm{km}$ of D1 in the direction to Ostrava. Time series from FCD and ASIM are shown in Figure 4. The figure shows an apparent disparity in the data between FCD and ASIM data. This is a very busy part of the D1 motorway. This FCD segment is placed between major motorway ramps. One ramp leads to the motorway to Vienna and the other one to motorway to Bratislava. The segment is approximately $2300 \mathrm{~m}$ long and ASIM sensor is installed $1400 \mathrm{~m}$ from the beginning of the segment.

There was short-term closure of the left lane realized on this segment on 8.3.2014. This meant a reduction in the number of lanes. Beginning of this short-term closure was on $195.2 \mathrm{~km}$ and end of this closure was on $195.6 \mathrm{~km}$, approximately 700 meters before the ASIM sensor. The short-term closure was implemented between 3:00 p.m. and 6:00 p.m. A traffic column was created in the front of the closure, which is visible in the FCD data in Figure 4. However, data from ASIM sensor show no traffic problem. This is because the column was created in front of traffic restrictions, but ASIM sensor is placed behind the restriction where the traffic flow is not restricted. Despite relative closeness of the incident to the ASIM sensor, it has failed to detect it. This situation confirms fact that to predict FCD time series from ASIM can be inaccurate. On the other hand, the FCD data source can be used to monitor the situation.

On 24. 3. 2014 at 9:00 at 112th $\mathrm{km}$ started the modernization of the D1 motorway. The repaired section started at 112 th $\mathrm{km}$ and ended at 104th $\mathrm{km}$ 


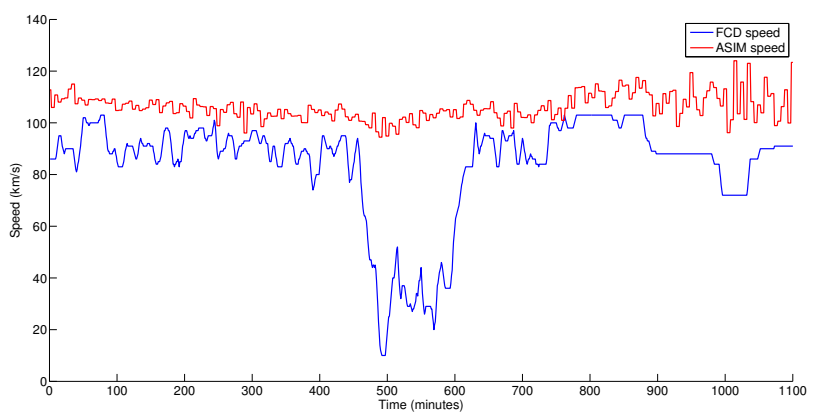

Fig. 4. Time series of speed from ASIM sensor 34.1 and corresponding FCD segment form 8. 3. 2014

in the direction to Prague. In the period between the 24.3. and 30.3. 2014, the traffic was restricted to one lane. Such traffic engineering measures at places with so intense traffic always cause problems and so it was in this case. Both traffic incidents with no causality happened during this period. Bottleneck, the place where two lanes were merging into one was placed at 112.5th $\mathrm{km}$. Beginning (or more exactly the end) of TMC segment was distanced approximately $3500 \mathrm{~m}$ against the road direction $(116 \mathrm{th} \mathrm{km})$ from the bottleneck and ASIM sensor was placed additional approximately 1500 meters from the beginning of the segment. This TMC segment was about 3500 meters long.

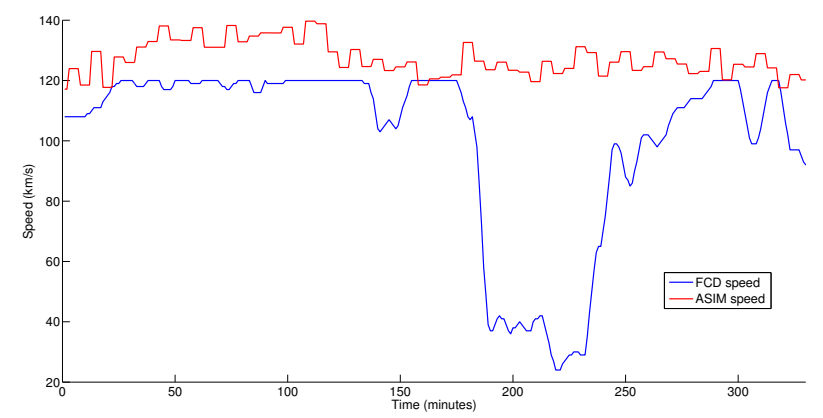

Fig. 5. Time series of speed from ASIM sensor 15.2 and corresponding FCD segment form 9:00 26. 3. 2014

The exported data from FCD and ASIM sensor show that in the first and the second day of reconstruction (24. 3. and 25. 3.) there were long traffic columns with length of more than $5 \mathrm{~km}$ almost all the day in the front of the merge. In those days, the speeds from FCD data and ASIM sensor were almost identical. 
This phenomenon was caused by the very rapid formation of traffic columns, which reached the ASIM sensor within minutes. The next day (26. 3.), it is already evident that after a series of media reports a certain percentage of drivers chose to postpone their trip, or chose another type of transport. This fact caused formation of shorter columns and their slower formation, which is evident in the difference between the speed of FCD and ASIM sensor (see Figure 5). Because the column has started reaching FCD segment, but has not reached the ASIM sensor, the speed of the FCD reduced, but the monitored detection sensor was still recording the speed of freely moving traffic flow. This is evident from the incident that happened on 26. 3. 2014 between 9:00 and 10:00 when the column intervened into the FCD segment, but has not reached the measured profile. In case of the next incident (14:00 on the same day), the column reached ASIM sensor yet.

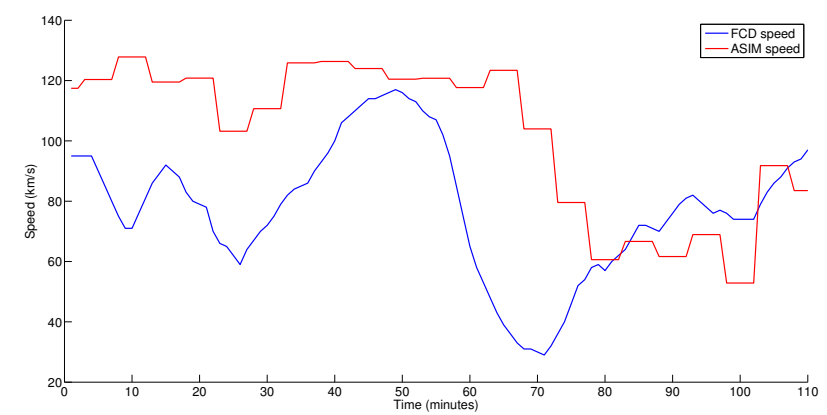

Fig. 6. Time series of speed from ASIM sensor 15.2 and corresponding FCD segment form 14:00 26. 3. 2014

From Figure 6 there is apparent delay in detection on the column by ASIM sensor. This is caused by slow formation of the column. The same is true in case of the dissolution of the column. The speed at first rises in ASIM sensor measurements and then gradually increases in FCD data. Only at the moment when the column does not interfere with the measured segment, traffic speeds again start to match. This again proves that prediction of FCD time series from ASIM is problematic due to the many specific configurations where profile sensor fails to detect traffic incident.

\section{Conclusion}

In this article, we have analysed Granger causality of speed time series from FCD and ASIM sensors with special focus on the traffic incidents. It was determined from the obtained results that in more than a half of incidents, there is Granger causality between FCD and ASIM data in both ways. In the other cases, there 
was only one way causality. It was mostly due to the fact, that when the incident happens some distance from the ASIM sensor, FCD is usually much swifter to record this incident than ASIM. Sometimes there is no causality at all between these time series but by thorough analysis of these incidents, we have proven that again this is caused by static nature of ASIM detectors which fails to detect some phenomena that can happen even in its close vicinity. Therefore, we can summarize that it is quite reliable to predict values of ASIM time series from FCD during the traffic incidents. Predicting FCD from ASIM can work most of the time too but is nowhere as reliable due to the nature of the sensor.

Results of this analysis underline importance of FCD data in Czech motorway traffic. ASIM sensors provide valuable information, but due to their sparsity are not able to describe accurately traffic in its entirety. They are failing especially when the traffic column is not long enough to reach the ASIM sensor or in the areas with many ramps. Due to this fact, it is very important to incorporate FCD data to any traffic model for Czech motorways. FCD-based analyses are very flexible, especially in an environment where it would be difficult (for economic or organizational reasons) to install dedicated static sensor infrastructure such as ASIM.

The intelligent combination of FCD with on-road sensors represents the perfect inputs to dynamic traffic models. New algorithms for data fusion will take benefit of the advantages and disadvantages of each technology, resulting in an optimal solution for traffic management problems.

In the near future, we are planning to find and implement methods for prediction in the analysed time series. These methods will be based on advanced approaches like Bayesian networks and Neural networks and will utilize results from this article.

\section{Acknowledgment}

This work was supported by the European Regional Development Fund in the IT4Innovations Centre of Excellence project (CZ.1.05/1.1.00/02.0070) and the national budget of the Czech Republic via the Research and Development for Innovations Operational Programme, by the project New creative teams in priorities of scientific research (reg. no. CZ.1.07/2.3.00/30.0055), supported by Op-

erational Programme Education for Competitiveness, and co-financed by the European Social Fund and supported by 'Transport Systems Development Centre' co-financed by Technology Agency of the Czech Republic (reg. no. TE01020155) and by the internal grant agency of VŠB Technical University of Ostrava, Czech Republic, under the project no. SP2015/114 'HPC Usage for Analysis of Uncertain Time Series'.

\section{References}

1. C. de Fabritiis, R. Ragona, and G. Valenti. Traffic estimation and prediction based on real time floating car data. In proceedings of 11th International IEEE Conference on Intelligent Transportation Systems, 2008. ITSC 2008., pages 197-203, 2008. 
2. D. Fedorčák, T. Kocyan, M. Hájek, D. Szturcová, and J. Martinovič. viarodos: Monitoring and visualisation of current trafficc situation on highways. In Proceedings of the 13th IFIP TC8 International Conference on Computer Information Systems and Industrial Management, CISIM 2014, 2014.

3. B. Ghosh, B. Basu, and M. O'Mahony. Multivariate short-term traffic flow forecasting using time-series analysis. Intelligent Transportation Systems, IEEE Transactions on, 10(2):246-254, 2009.

4. C. Granger. Some recent development in a concept of causality. Journal of Econometrics, 39(1-2):199-211, 1988.

5. A. Graser, M. Dragaschnig, W. Ponweiser, H. Koller, M.-S. Marcinek, and P. Widhalm. Fcd in the real world - system capabilities and applications. In proceedings of 19th ITS World Congress, Vienna, Austria, page 7, 2012.

6. M. Jones, Y. Geng, D. Nikovski, and T. Hirata. Predicting link travel times from floating car data. In Proceedings of International IEEE Conference on Intelligent Transport Systems (ITSC), 2013.

7. R.-A.-I. Khan, B. Landfeldt, and A. Dhamdher. Predicting travel times in dense and highly varying road traffic networks using starima models. Technical report, School of Information Technologies, The University of Sydney and National ICT Australia, 2012.

8. G. Kuhns, R. Ebendt, P. Wagner, A. Sohr, and E. Brockfeld. Self evaluation of floating car data based on travel times from actual vehicle trajectories. In IEEE Forum on Integrated and Sustainable Transportation Systems, 2011.

9. M. Li, Y. Zhang, and W. Wang. Analysis of congestion points based on probe car data. In Proceedings of International IEEE Conference on Intelligent Transportation Systems, ITSC '09, pages 1-5, 2009.

10. Y. Ma, H. J. van Zuylen, and J. van Dalen. Freight origin-destination matrix estimation based on multiple data sources: Methodological study. In TRB 2012 Annual Meeting, 2012.

11. D. S. McLeod, L. Elefteriadou, and L. Jin. Travel time reliability as a performance measure: Applying florida's predictive model on the state's freeway system. In TRB 2012 Annual Meeting, 2012.

12. R.-P. Schăfer, K.-U. Thiessenhusen, and P. Wagner. A traffic information system by means of real-time floating-car data. In Proceedings of ITS World Congress 2002, Chicago, USA, 2002.

13. E. I. Vlahogianni. Enhancing predictions in signalized arterials with information on short-term traffic flow dynamics. Journal of Intelligent Transportation Systems, 13(2):73-84, 2009.

14. P. Widhalm, K. H., and P. W. Identifying faulty traffic detectors with floating car data. In IEEE Forum on Integrated and Sustainable Transportation System, 2011.

15. Y.-J. Wu, F. Chen, C.-T. Lu, and B. Smith. Traffic flow prediction for urban network using spatio-temporal random effects model. In 91st Annual Meeting of the Transportation Research Board, 2011.

16. W. Zeng, Z. He, R. Lu, Zhuang, Lijiang, and X. Xia. Freeway segment speed estimation model based on distribution features of floating-car data. In TRB 2012 Annual Meeting, 2012.

17. J. Zheng. Road travel time estimation with gps floating car data. In Stander Symposium Posters. Book 191, 2012. 